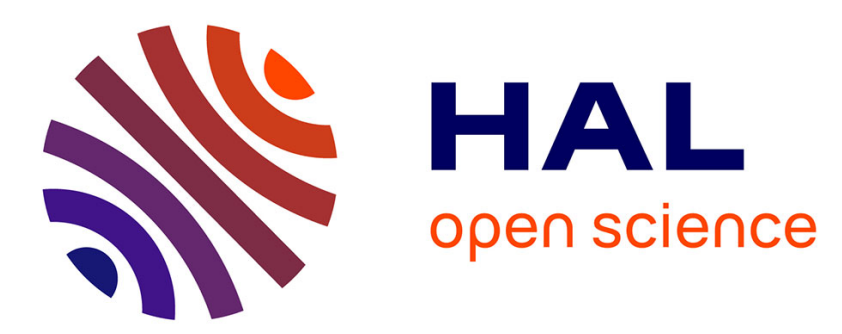

\title{
Influence of a bow of finite width on bowed string motion : numerical modelling and experimental evidence
}

\author{
R. Pitteroff, J. Woodhouse
}

\section{To cite this version:}

R. Pitteroff, J. Woodhouse. Influence of a bow of finite width on bowed string motion: numerical modelling and experimental evidence. Journal de Physique IV Proceedings, 1994, 04 (C5), pp.C5-605C5-608. 10.1051/jp4:19945129 . jpa-00252805

\section{HAL Id: jpa-00252805 https://hal.science/jpa-00252805}

Submitted on 1 Jan 1994

HAL is a multi-disciplinary open access archive for the deposit and dissemination of scientific research documents, whether they are published or not. The documents may come from teaching and research institutions in France or abroad, or from public or private research centers.
L'archive ouverte pluridisciplinaire HAL, est destinée au dépôt et à la diffusion de documents scientifiques de niveau recherche, publiés ou non, émanant des établissements d'enseignement et de recherche français ou étrangers, des laboratoires publics ou privés. 


\title{
Influence of a bow of finite width on bowed string motion: numerical modelling and experimental evidence
}

\author{
R. PITTEROFF and J. WOODHOUSE
}

University of Cambridge, Dept. of Engineering, Trumpington Street, Cambridge CB2 1PZ, U.K.

\begin{abstract}
An enhanced physical model of the bowed string presented previously [1] is explored. It takes into account: the width of the bow, the angular motion of the string, bow-hair elasticity and string bending stiffness. The results of an analytical investigation of a model system - an infinite string sticking to a bow of finite width and driven on one side of the bow - are compared with experimental results published by Cremer [2] and reinterpreted here. Comparison shows that both the width of the bow and the bow-hair elasticity have a large impact on the reflection and transmission behaviour. In general, bending stiffness plays a minor role. Furthermore, a method of numerical simulation of the stiff string bowed with a bow of finite width is presented along with some preliminary results.
\end{abstract}

\section{INTRODUCTION}

The aim of the research described here is an improved simulation model of the bowed string allowing for a bow of finite width, for bow-hair elasticity (that is longitudinal bow-hair stretching), and for the string's bending stiffness. In Section 2 the mechanical model for the contact area of bow and string is briefly described. In a second step this contact area is linked and matched to two semi-infinite sections of free string. Section 3 deals with the reflection and transmission behaviour of this system, where incident sinusoidal waves travel towards the bow and interact with it. The theoretical model is used to shed some light on results obtained by Cremer [2] and Eisenberg [3] in an experimental investigation of this system. Finally, the physical model of the contact area is inserted into a numerical simulation of a string of finite length.

\section{MODELLING OF THE CONTACT AREA}

In the contact area of bow and string the surface of the string is subjected to a tangential frictional force distributed across the width of the bow. The two governing equations for this string segment are the equations of momentum and angular momentum, found to be ordinary wave equations. The two wave equations are coupled through the force term common to both. This force also leads to deformation of the compliant bow-hair. The bow-hair is thought of as a massless support of linear elastic behaviour and velocity-proportional damping. Tests on individual bow-hairs support this approximation of the material behaviour. The force-deformation relation provides a third equation. (In this context forces between bowhairs shall not be taken into account although they certainly have non-negligible influence in terms of energy losses.)

For the sticking/rolling regime the equations of momentum, the force-deformation relation and the equation expressing the rolling constraint result in a system of linear equations that can be solved analytically. Slipping (when the rolling constraint no longer holds) requires a further equation; it is a nonlinear friction law relating the frictional force to the relative velocity of bow and string. Any solution of the "slipping" system of equations must resort to numerical methods.

\section{AN 'INFINITE' STRING STICKING/ROLLING ON A BOW}

A simple experimental method for gaining some insight into the mechanics of the contact area of string and bow - a method of the inverse scattering type - is described by Cremer [2]: a bow is placed on a very long string and a bow force sufficiently high to prevent slipping is applied. Wave packets (sinusoidal) are generated on the free string and travel to meet and interact with the bow. Incident, reflected and 
transmitted waves are monitored with a simple electro-inductive device. Cremer's student Eisenberg [3] carried out a number of experiments with various string and bow materials. As mentioned above this problem lends itself to an analytical approach. Cremer [2] used a model assuming an infinite string sticking/rolling on a rigid point bow to explain the experimental results. He concluded that the reflection and transmission behaviour of the studied system is largely determined by the string's bending stiffness. Cremer [2] chose to publish the results from an experimental set-up using a violin bow and a nylon string of $3 \mathrm{~mm}$ diameter. Figure 1a shows these results and his theoretical prediction. The agreement seems to be satisfactory. This good fit was achieved, however, by setting the Young's modulus of the string to a value one order of magnitude higher than that measured [3].

This current work carries on from Cremer's theoretical approach by introducing the finite bow: this means joining the one dimensional continuum of the string sticking/rolling on the bow - described through the mechanical model summarised above - to sections of free string on either side, and establishing the correct boundary conditions at the interfaces. Adding the bow width to Cremer's model leads to considerably different results. Figure $1 \mathrm{~b}$ displays the prediction from a model assuming a finite rigid bow, the first natural improvement of Cremer's rigid point bow model. Figure $1 \mathrm{~b}$ again features Cremer's prediction, but now both calculations use the true value of the Young's modulus of the string. Clearly a rigid bow model, be it a point or a finite bow, fails to describe the system behaviour accurately.

Allowing for bow-hair compliance (Figure 1c) leads to a better model. The Young's modulus of the bow-hair is set to the measured value of $7.5 \mathrm{GPa}$. It is furthermore assumed that only one layer of bow-hair interacts with the string. This model can be improved further by taking into account bow hair damping (Figure 1d). Agreement with the experimental results is fairly good.
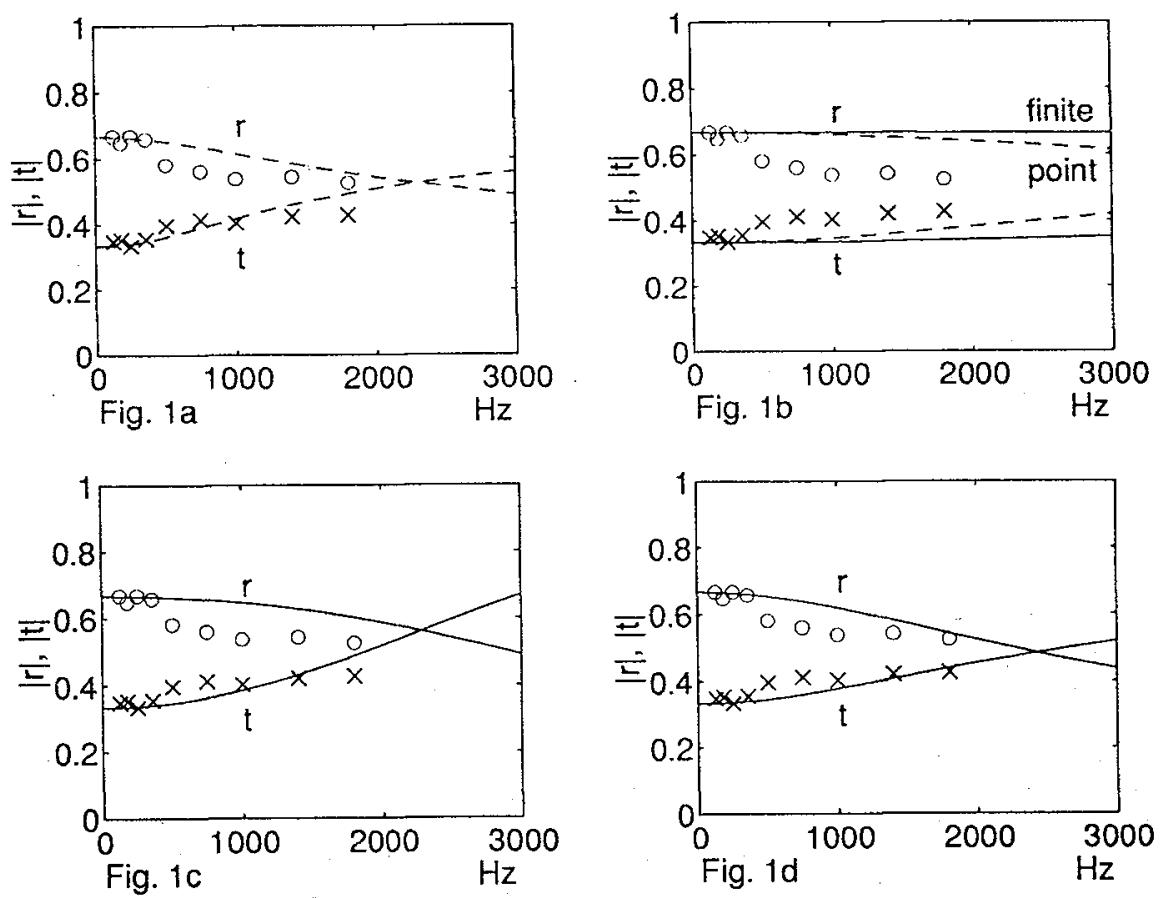

Figures 1a-d: Reflection and transmission coefficients from experimental and theoretical investigation. a: Measured (o) reflection and $(x)$ transmission coefficients, $(-)$ Cremer's rigid point bow model with adjusted Young's modulus. b: (--) Cremer's rigid point bow model and $(-)$ rigid finite bow model, both with the true value of the Young's modulus. $\mathrm{c}$ : $(\rightarrow)$ compliant finite bow model. d: (-) compliant finite bow model with velocity-proportional bow-hair damping.

By arbitrarily lowering the theoretical number of bow hairs in contact with the string even closer fits can be achieved. The numerical modelling of a bowed string described below reveals that it often happens that only a part of the bow-hair is sticking while the rest is slipping. For a more accurate quantitative investigation it would be beneficial to develop means of establishing the actual number of hairs in contact 
with the string. The question of the extent to which layers of hair not in direct contact with the string come into play could also be addressed.

\section{NUMERICAL SIMULATION OF THE BOWED STIFF STRING}

The non-linear friction curve which comes into play when the string slips on the bow-hairs calls for numerical integration methods. Time domain approaches have proven to be most useful in modelling of the bowed string. The simplest method, an explicit time stepping finite difference method, is also the one which gives us the most insight into physical processes. In the numerical model of the flexible bowed string presented in [1] displacements and force in the contact area are calculated through the discretisation of the equations described in Section 2. The rest of the string and the terminations are modelled using the analytical methods described by McIntyre et al [4], i.e. simple wave propagation on the free string and convolution of the string displacement values with reflection functions to model the effect of the string's terminations. The finite difference scheme reaches somewhat beyond the contact area into the free string, far enough to allow for modelling of the boundary conditions. The boundary conditions are jump conditions which can be transcribed directly into finite difference equations.

To include bending stiffness, the numerical method must be upgraded in several ways. Firstly, the boundary conditions at the interfaces of free string and bow can only be fulfilled by taking the near fields on either side of the bow into account. The finite difference scheme must therefore be extended into the free string to encompass the exponentially decaying evanescent waves generated at the interfaces. Typically an extension of the computational domain by a bow-width is sufficient. Secondly, to ensure numerical stability the boundary conditions at the edge of the contact area are no longer explicitly translated into finite difference equations. They are now satisfied by means of a continuous finite difference scheme where coefficients change at the interfaces. Also for reasons of numerical stability, the boundary conditions used to match the numerically described domain with the outer analytical solution now assume an ideal flexible string at the boundary of the computational domain.
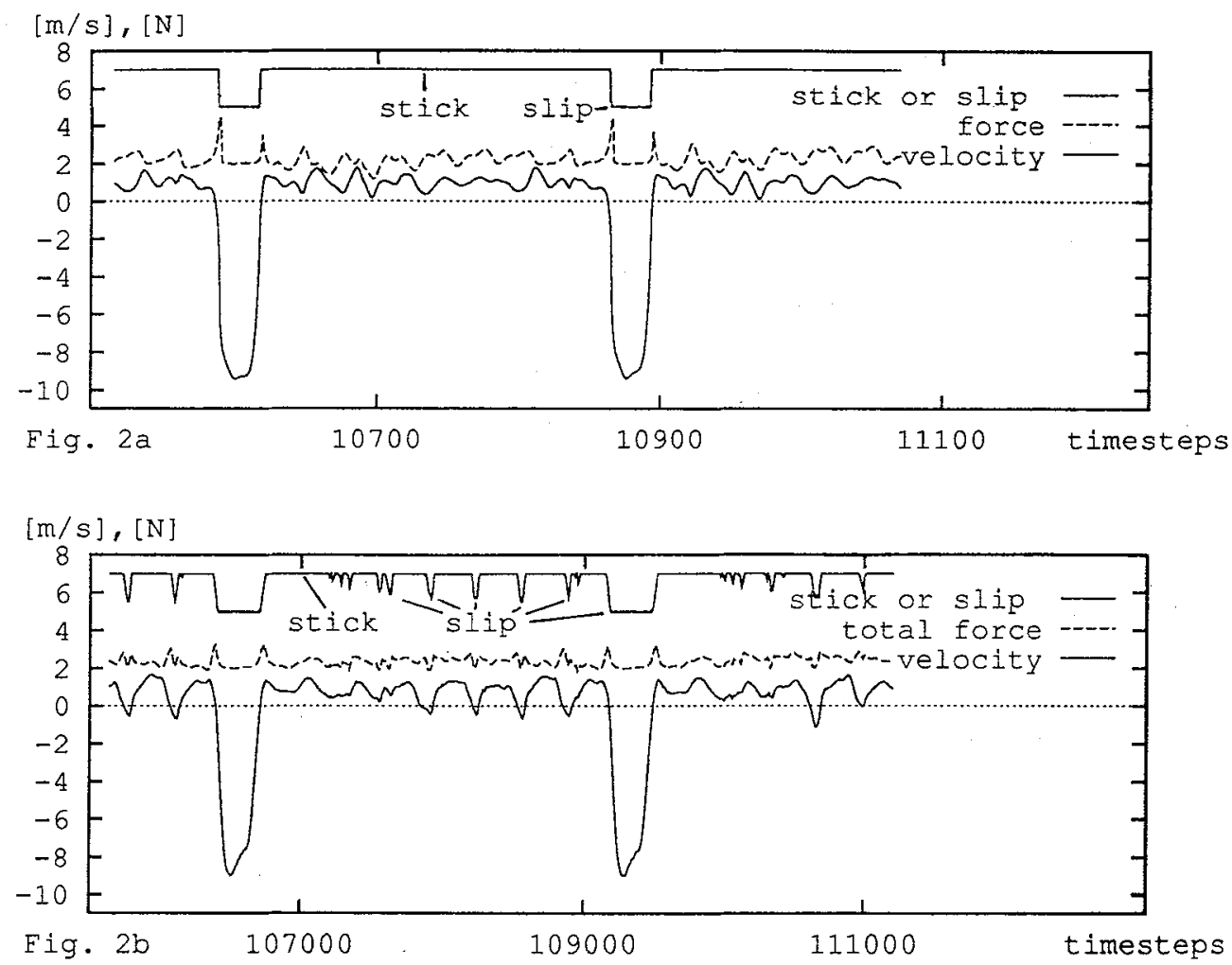

Figure 2a-b: a: Numerical simulation of a flexible bowed string assuming a point bow: string centre-line velocity and frictional force over time. $b$ : Numerical simulation of a stiff bowed string assuming a finite bow: string centre-line velocity at the edge of the bow facing the bridge and total frictional force over time. In both figures the top, solid line indicales stick (high value) and slip (low value). The finite bow features intermediate states. 
Thirdly, the waves travelling to the string's terminations and back should suffer dispersion due to bending stiffness. This effect can be modelled through a suitable choice of reflection functions (described by Woodhouse [5]). In the example presented here, however, the applied reflection functions are simply Gaussians so that bending stiffness is only taken into account near the bow.

The results presented in Figures $2 \mathrm{a}-\mathrm{b}$ are from runs of simulation programs initialised with the ideal Helmholtz sawtooth waveform. Figure $2 \mathrm{a}$ represents a flexible-string, point-bow model and Figure $2 \mathrm{~b}$ a stiff-string, finite-bow model. Both figures display the centreline velocity of the string and the frictional force. For the finite bow the force value is the integral of the distributed force across the bow. The solid line at the top of the graphs indicates sticking and slipping and, for the finite bow model, the transition between the two states.

The cycles shown (periods 39 and 40) are taken during the system's stationary regime. The model string approximates a Pirastro Eudoxa A-string of $0.68 \mathrm{~mm}$ diameter, $0.3 \mathrm{~m}$ length tuned to $440 \mathrm{~Hz}$. The bow is taken to be $10 \mathrm{~mm}$ wide. The Young's modulus of the bow-hair is set to the measured value of 7.5 $\mathrm{GPa}$ and a moderate value of bow-hair damping is chosen. In order to mark the difference between the displayed "flexible" and "stiff" runs of the simulation programs a rather high value was chosen for the Young's modulus of the string $(20 \mathrm{GPa})$. The centre of the bow divides the string in the ratio 15:124. The bow speed is $1 \mathrm{~m} / \mathrm{s}$ and the normal force equals $5 \mathrm{~N}$. The torsional phase velocity is 2.5 times the transverse, the admittance ratio is 0.617 . One can think of a point bow as simply being composed of fewer bow-hairs or as the entire ribbon of bow-hair confined to one point. Here, the latter is assumed to allow for a comparison of models rather than bows.

The most obvious difference between the two runs are the intermittent partial slips during sticking on the finite bow. The frictional force during sticking is highest on the side of the bow facing the bridge. This has to do with the fact that the bow attempts to move the entire section of the string sticking to it with a constant velocity, which is incompatible with the kinematics of the Helmholtz motion. Peaks in frictional force are followed by partial slips on the bridge side of the bow that allow the segment of string under the bow to straighten out somewhat. This produces drops in the string centre-line velocity and affects the wave travelling to the bridge. The waveforms and the harmonic content of the waves travelling to the bridge differ strongly from one model to another.

Release and recapture of the string are not instantaneous for the finite bow. Typically both transitions take $1.5 \%$ of a period. The duration of ubiquitous slipping does not vary much from one model to another. The maximum amplitude of the slip velocity tends to be higher for point-bow models.

\section{CONCLUSIONS}

For the linear problem of a string sticking/rolling on a bow, the proposed mechanical model of the contact area compares well with experimental results. The finite width of the bow, the bow-hair compliance and damping seem to be important ingredients of any such model. A family of simulation algorithms modelling flexible and stiff strings bowed with point and finite bows has been developed. In future work these models will be studied in an attempt to gain more insight into the effect of the width of the bow and the bending stiffness on the playability of model systems of the bowed string.

\section{References}

[1] Pitteroff R., "Modelling of the bowed string taking into account the width of the bow", SMAC 93, Stockholm 28 July - 1 August 1993 (submitted).

[2] Cremer L., "The physics of the violin" (MIT Press, Cambridge MA, U.S.A.,1985).

[3] Eisenberg P., Studienarbeit (project report) (TU Berlin, 1967).

[4] McIntyre M. E., Schumacher R. T. \& Woodhouse J., J. Acoust. Soc. Am. 74 (1983) 1325-1345.

[5] Woodhouse J., Acustica 78 (1993) 125-153. 